\title{
Analysis and Recognition of Walking Movements*
}

\author{
James W. Davis Stephanie R. Taylor \\ Dept. of Computer and Information Science \\ Ohio State University \\ Columbus, OH 43210 USA \\ \{jwdavis, taylors\}@cis.ohio-state.edu
}

\begin{abstract}
We present an approach for recognizing human walking movements using low-level motion regularities and constraints. Biomechanical features for classification are automatically extracted from video sequences of walkers. A multiplicative classification rule using statistical distances is then used to determine whether an unknown motion is consistent with normal walking patterns. Recognition results are shown distinguishing walking examples across multiple speeds from other non-walking locomotions.
\end{abstract}

\section{Introduction}

We can immediately recognize an atypical walking pattern (e.g., limping) from inconsistencies of that motion with our strong perceptual category for normal human walking. Furthermore, the normal walking category itself contains variations (e.g., stride length increases with walking speed [8]) which do not affect the classification. Most approaches to motion recognition have overlooked the dynamic changes exhibited within a motion category, having only examined a small number of walkers each moving at a single pace.

In this paper, we describe a motion-based method for distinguishing normal walking movements at multiple speeds from other atypical or non-walking locomotions. By modelling low-level dynamic regularities and constraints in normal walking patterns, a sub-space of human walking motions is constructed for classification. Such a system would be particularly useful for automated visual surveillance and monitoring systems in helping to identify people that may be injured or that may require assistance by recognizing their atypical motion patterns.

We begin with a review of related research (Sect. 2) and briefly describe the underlying motion representation (Sect.

\footnotetext{
* Appears in International Conference on Pattern Recognition, Quebec City, Canada, August 11-15, 2002, pp. 315-318.
}

3). The method used to track the person (Sect. 4) and the motion features used to characterize the walking gait are then described (Sects. 5-6). We lastly present the recognition method and results (Sects. 7-8), and conclude with a summary of the research (Sect. 9).

\section{Related Work on Recognizing Gait}

The most common approach to recognition of gait is the analysis of trajectory information derived from features of the walking body. The frequency-based approach of [9] examined the phase relationships of periodic elements for the task of person identification from walking. Also addressing identification, [10] used the spatio-temporal braided patterns of the legs within the XYT volume to detect walking, and then extracted features of the pattern to identify individuals. To distinguish children from adults, [5] used correlated stride-based properties of their spatio-temporal walking styles for classification. Additionally, [2] incorporated a sequence of body signature skeletons into an HMM framework to determine a posture transition path for recognition.

Certain types of motion recognition can benefit from a model-based tracking pre-processor, but there are situations where direct motion recognition with no part tracking is applicable (e.g., [4]). In [11], the periodic motion of pixels (as created from walking) was analyzed throughout a sequence using Fourier techniques. Another image-based periodicity approach is described in [3], where time-frequency analysis of a self-similarity measure between images in a sequence was used to detect and characterize periodic (walking) motion. In [7], individual walkers were recognized by combining an Eigenspace transformation and a Fisher Linear Discriminate function on background-subtracted silhouettes.

Our interests focus on the categorization of motion to classify the type of movement. We present a low-level approach to distinguish walking from other non-walking locomotions. 


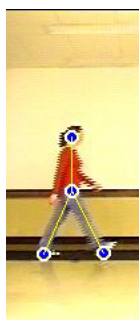

(a)

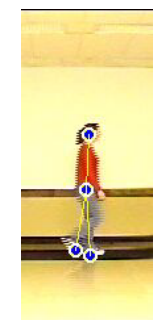

(b)

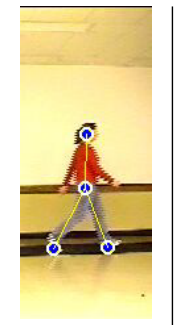

(c)

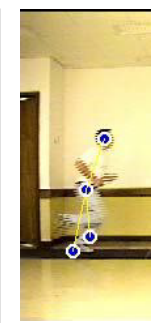

(d)

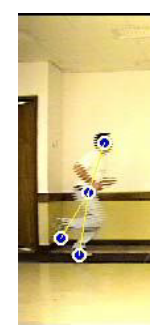

(e)
Figure 1. Person tracking in video. (a)-(c) Automatic identification of head, waist, and feet. (d) Tracking of runner without rotation step. (e) Tracking of runner with rotation step.

\section{Motion Category Representation}

Our overall goal is to represent various types of human motion with low-level categories to permit efficient recognition from partial (or limited) visual input. We define a motion category as a parameterization of dynamic regularities together with any constraints:

\section{Motion Category $=$ Dynamic Regularities + Constraints}

The smoothly varying regularities describe the "genericity" (or acceptable variations) of the class. The structural constraints allow for any fixed values to be included in the representation. Multiple categories are separated by discontinuities in the regularity and constraint parameterizations.

To illustrate a simple motion category, consider the class of pendular motion for a swinging particle at the end of a light inextensible cord. The structural constraints include the choices for the particle mass $M$, cord length $L$, and gravity $G$. The dynamic regularity (for small amplitudes) consists of the correlation of the period of movement $T$ to $L / G$, as defined by $T=2 \pi \sqrt{L / G}$. We can then predict (or verify) the period of movement $T$ for a pendulum from the observed length of the cord $L$ (mass does not effect the period). We seek to categorize human motion in a similar manner to classify different types of movements from a small number of correlated features and constraints.

\section{Person Tracking in Video}

To track a person in video, we use a method based on the W4 approach [6] to locate the head, waist, and feet. The method is best suited to fronto-parallel views of the walker, but can accommodate slightly different viewpoints. We begin by extracting the person's silhouette in each video frame with a standard background-subtraction technique using RGB pixel differences, dilation, and removal of small pixel regions. Then, we fit a line oriented to the principal axis of the silhouette and un-rotate the silhouette so that the principal axis is vertical. After applying a bounding box to the un-rotated silhouette, we vertically separate the head, torso, and leg regions using average anatomical proportions.

The head point is found as the centroid of the silhouette pixels in the head region. The waist location within the torso region is determined from the mean $\mathrm{x}$-value of the silhouette pixels in the torso region and the expected y-coordinate of the waistline. To locate the feet, the region of the bounding box below the waistline is divided into equal left and right halves, where each half is assumed to contain one leg. Within each leg region, the principal axis of the silhouette pixels within that region is found. The foot points are determined to be the most extreme silhouette pixels in the direction of those lines away from the waist. The data points are then rotated back to their actual positions in the image, low-pass filtered, and stabilized (relative to the head point). Results of the tracking method are shown in Fig. 1.(a)-(c).

We added the rotation step to the W4 method in order to increase the likelihood of finding all the necessary body parts, even when the person's body is oriented so that both legs are in the same half of the leg region of the bounding box. Figure 1.(d)-(e) compare the body tracking method of a runner with and without the rotation step.

\section{Low-Level Gait Features}

Rather than attempting to match joint angles, limb lengths, or poses, we employ four motion properties of the tracked feet locations to demonstrate the approach. Three features together comprise the dynamic regularities; the remaining feature is a structural constraint. Additional features showing biomechanical regularities and constraints in locomotion to extend the model can be found in [8].

\subsection{Dynamic Regularity Features}

The first three biomechanical features are temporal properties and are generally independent of the camera position.

- Cycle Time: $T_{c}$. One of the most fundamental descriptors of locomotion is the cycle time of a leg, which decreases with increasing walking speed. Cycle time is determined by calculating the time difference between neighboring minimal (or maximal) peaks in each foot's $\mathrm{x}$-trajectory.

- Stance/Swing Ratio: $\tau$. Our second feature is the ratio of stance and swing times of a leg. The stance time is approximated by the front-to-back interval of the leg, and corresponds to the time difference between a maximal peak and the subsequent minimal peak in 


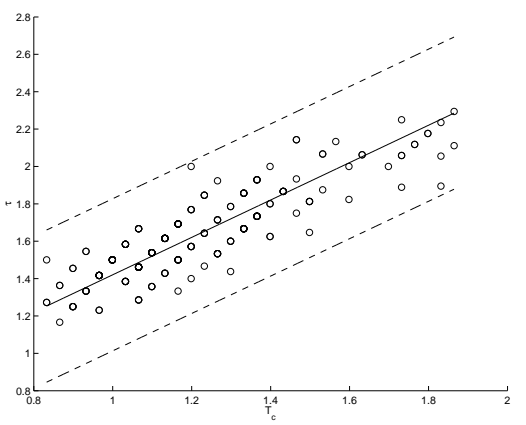

(a)

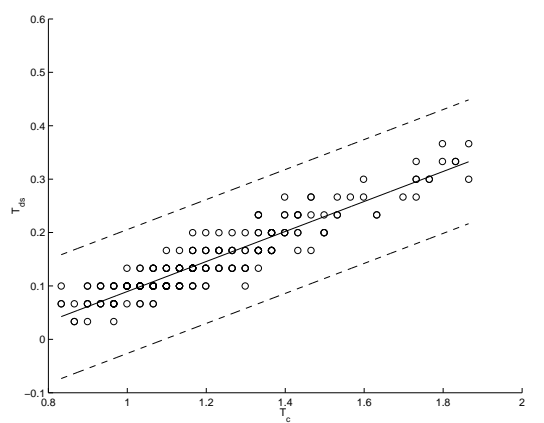

(b)

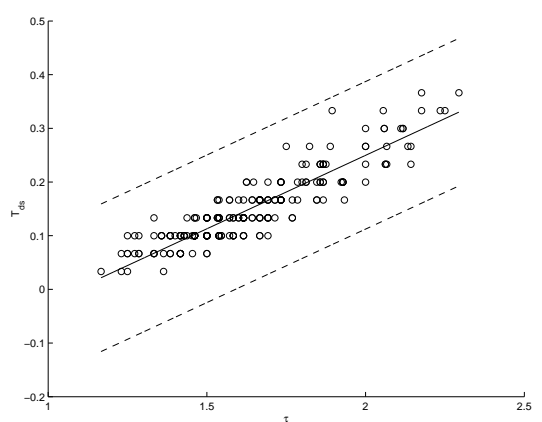

(c)

Figure 2. Dynamic feature regularities. (a) $T_{c}$ vs. $\tau$. (b) $T_{c}$ vs. $T_{d s}$. (c) $\tau$ vs. $T_{d s}$. Linear prototypes with $\pm 5 \sigma$ are shown.

the $\mathrm{x}$-trajectory. Similarly, the swing time is approximated by the temporal interval of the back-to-front translation of the leg, and is determined by calculating the time difference between a minimal peak and the subsequent maximal peak in the corresponding foot's $\mathrm{x}$-trajectory. The stance/swing ratio decreases as a person walks faster.

- Double Support Time: $T_{d s}$. Our next feature approximates the time that both feet are in the stance phase (double support), and is measured by the time difference from when one leg begins its return (onset of stance time in leg 1) to when the other leg starts moving forward (onset of swing time in leg 2). The double support time is determined by the temporal difference between a minimal peak in one foot's x-trajectory and the nearest maximal peak in the other foot's $x-$ trajectory. The double support interval approaches zero as a person walks faster.

\subsection{Constraints}

In this paper we include only one view-based constraint, but other physical or motion constraints could be added.

- Extension Angle: $\theta$. The extension angle is the fronto-parallel angle made from the xy position of the maximal forward extension of the leg in front of the body to the most distant extension of the leg behind the body. We calculate this feature for a given foot as the angle between the xy coordinates of maximal spatiotemporal curvature in the front and back halves of the xy-trajectory for a single walking cycle. This angle is nearly zero for people during walking.

\section{Walking Category}

To construct the motion category for walking, we recorded a video database of 17 male and female walkers each moving at slow, medium, and fast paces. From the person tracking output, we computed our motion features $\left(T_{c}, \tau, T_{d s}, \theta\right)$ for multiple cycles of each individual. Each walking cycle was analyzed separately with no averaging of features over multiple cycles. Each leg was also analyzed separately.

Three pairwise combinations of the temporal features were used to construct the dynamic regularities. In Fig. 2.a, we present $T_{c}$ vs. $\tau$ that characterizes the typical walking timings for a single leg (correlation of $\rho=0.87$ ). In Fig. 2.b, $T_{c}$ vs. $T_{d s}$ represents how the two legs move in temporal relation to one another over multiple speeds $(\rho=0.93)$. Lastly in Fig. 2.c, we see $\tau$ vs. $T_{d s}$ that also relates to the temporal movement of the two legs $(\rho=0.91)$. The constraint $\theta$ had a nearly Gaussian distribution centered around zero degrees $\left(\sigma_{\theta}=1.392\right)$.

\section{Recognition Method}

To determine class membership for a new movement to this walking category, statistical measures of the new features are combined into a single binary classification result.

For the regularity features, the perpendicular Mahalanobis distance of the new feature values to linear prototypes are computed using

$$
R_{i}=\frac{\left|\alpha_{i} X_{i}-Y_{i}+\beta_{i}\right|}{\sigma_{i} \sqrt{\alpha_{i}^{2}+1}}
$$

with $\left\{X_{1}=T_{c}, Y_{1}=\tau, \alpha_{1}=1.000, \beta_{1}=.420\right\}$ for the single-leg regularity, and $\left\{X_{2}=T_{c}, Y_{2}=T_{d s}, \alpha_{2}=.281\right.$, $\left.\beta_{2}=-.191\right\}$ and $\left\{X_{3}=\tau, Y_{3}=T_{d s}, \alpha_{3}=.274\right.$, 
$\left.\beta_{3}=-.298\right\}$ for the coupled-legs regularities. The $\alpha_{i}, \beta_{i}$ parameters were determined from training using an Eigenvector line fitting process that minimizes the sum of squares of the perpendicular distances from the training points to the linear prototype. The standard deviations $\sigma_{i}$ were computed as the overall deviation along each regularity $\left(\sigma_{1}=.081\right.$, $\sigma_{2}=.023, \sigma_{3}=.027$ ). We show the linear prototype models with $\pm 5 \sigma$ class boundaries in Fig. 2.

Constraint features are examined using the standard Mahalanobis distance. The distance for a new $\theta$ to the model is computed by

$$
C_{\theta}=\frac{|\theta-\bar{\theta}|}{\sigma_{\theta}}
$$

with $\bar{\theta}=.064$ and $\sigma_{\theta}=1.392$ degrees.

To be classified as typical walking, the regularity and constraint distances $\left(R_{i}, C_{\theta}\right)$ are thresholded and incorporated into the binary result $M$ using a product classification rule to discount those movements having any nonconforming properties:

$$
M=\hat{C}_{\theta} \prod_{i} \hat{R}_{i}, \quad \hat{X}_{k}= \begin{cases}1 & X_{k} \leq 5 \\ 0 & \text { otherwise }\end{cases}
$$

The result is a single binary membership assignment for the movement to the walking category $(0=$ reject, $1=$ accept $)$.

\section{Experimental Results}

To test the approach, we collected several new examples of normal walking and other non-walking locomotion sequences. The set of typical walking included slow/medium/fast paces of one person filmed a week apart from the training sequences and of three new people not used in training. Additionally, a single sequence from the walking dataset of [1] and of a 4-year-old child were tested. We also tested an outdoor sequence in which the walker moved at a non-fronto-parallel angle relative to the camera. The non-walking locomotions included video sequences of people jogging, running, limping, skipping, marching (two styles), crawling, sidestepping, walking backwards, hopping, and tumbling. We also tested two artificial walking sequences: a fast pace slowed down by $50 \%$ and a slow pace played at double the normal speed.

The classification results using the gait features and Eqn. 3 were calculated for the sequences. All 15 normal walking sequences were correctly classified (with most $R_{i}, C_{\theta}$ within $2.5 \sigma$ ). Eleven of the 14 non-walking sequences were correctly rejected (most with at least one regularity or constraint distance $>8 \sigma$ ).

The three incorrectly classified sequences were skipping, high-knee marching, and walking backwards. Skipping and high-knee marching should have had invalid extension angles since the foot trajectories peak high near the body.
However, imprecise foot tracking when the legs are close together resulted in inaccurate spatio-temporal curvature calculations, so these sequences were misclassified. We hypothesized that walking backwards would be classified as non-walking due to different stance/swing ratios or extension angles. However, the temporal and spatial feature values are almost identical to those of people walking forward.

\section{Summary}

We presented an approach for representing and recognizing walking movements using a small number of low-level motion regularities and constraints. To construct the walking category, biomechanical motion features were computed from real video sequences of 17 people walking at multiple speeds. A multiplicative classification rule using statistical distances was used to determine whether an unknown motion was consistent with normal walking patterns. Results using several normal walking patterns and non-walking locomotions demonstrated the ability of the approach.

\section{References}

[1] A. Baumberg and D. Hogg. Learning flexible models from image sequences. In Proc. Euro. Conf. Comp. Vis., pages 299-308, 1994.

[2] I. Chang and C. Huang. The model-based human body motion analysis system. Image and Vision Comp., 18(14):1067-1083, 2000.

[3] R. Cutler and L. Davis. Robust real-time periodic motion detection, analysis, and applications. IEEE Trans. Patt. Analy. and Mach. Intell., 22(8):781-796, 2000.

[4] J. Davis and A. Bobick. The representation and recognition of action using temporal templates. In Proc. Comp. Vis. and Pattern Rec., pages 928-934. IEEE, 1997.

[5] J. Davis. Visual categorization of children and adult walking styles. In Proc. Int. Conf. Audio- and Video-based Biometric Person Authentication, pages 295-300, 2001.

[6] I. Haritaoglu, D. Harwood, and L. Davis. W4: Who? When? Where? What? A real time system for detecting and tracking people. In Proc. Int. Conf. Auto. Face and Gesture Recog., pages 222-227, 1998.

[7] P. Huang, C. Harris, and M. Nixon. Recognising humans by gait via parametric canonical space. Artif. Intell. in Eng., 13:359-366, 1999.

[8] V. Inman, H. Ralston, and F. Todd. Human Walking. Williams \& Wilkins, Baltimore, 1981.

[9] J. Little and J. Boyd. Recognizing people by their gait: the shape of motion. Videre, 1(2):2-32, 1998.

[10] S. Niyogi and E. Adelson. Analyzing and recognizing walking figures in XYT. In Proc. Comp. Vis. and Pattern Rec., pages 469-474. IEEE, 1994.

[11] R. Polana and R. Nelson. Low level recognition of human motion. In Workshop on Motion of Nonrigid and Articulated Objects, pages 77-82. IEEE Computer Society, 1994. 